\title{
Hangst, Matthias (2015): Regionalplanung im Bundesstaat. Die Rechtsstellung der Träger der Regionalplanung seit der Föderalismusreform I und dem Raumordnungsgesetz 2009 unter besonderer Berücksichtigung Baden-Württembergs
}

\author{
Berlin: Duncker \& Humblot. = Schriften zum Öffentlichen Recht, Band 1306, 558 S.
}

\section{Susan Grotefels ${ }^{1}$}

Online publiziert: 26. September 2016

(c) Springer-Verlag Berlin Heidelberg 2016

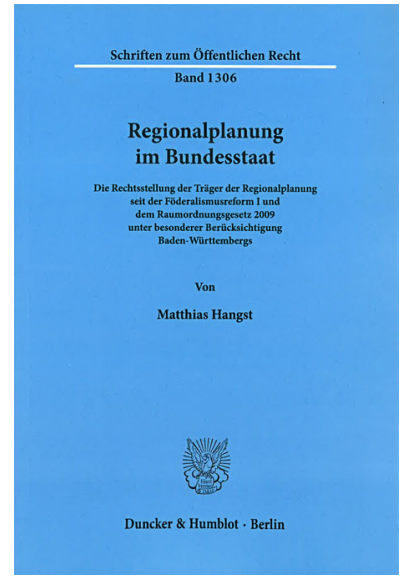

Seitdem die Raumordnung 2006 durch die Föderalismusreform I zum Gegenstand der konkurrierenden Gesetzgebungszuständigkeit des Bundes mit der Möglichkeit zur Abweichungsgesetzgebung durch die Länder verfassungsrechtlich neu aufgestellt wurde, setzt sich die rechtswissenschaftliche Forschung weitaus intensiver mit der zusammenfassenden überörtlichen und fachübergreifenden Raumordnungsplanung auseinander. Spätestens seit Erlass des Raumordnungsgesetzes 2009 durch den Bund und die daraufhin folgenden Landesplanungsgesetze wird eine Reihe neuer interessanter Fragestellungen um das Kompetenzgefüge zwischen Bund und Ländern aufgeworfen, die allerdings bei genauerer Betrachtung den früheren Problemen

Dr. Susan Grotefels

grotefe@uni-muenster.de

1 Zentralinstitut für Raumplanung,

Westfälische Wilhelms-Universität Münster,

Wilmergasse 12-13, 48143 Münster, Deutschland mit dem Verständnis der Rahmengesetzgebungskompetenz und ihrer Grenzen bisweilen sehr ähneln.

In der Planungspraxis und Politik spielt die Raumordnungsplanung in Form von Landesentwicklungsplänen oder -programmen sowie von Regionalplänen eine zunehmend wichtige Rolle, da sie beispielsweise zur Umsetzung der Energiewende, zur Einzelhandelssteuerung oder zur Verminderung des Flächenverbrauchs ihren Beitrag leisten soll. Der Titel der Arbeit macht bereits eindeutig klar, dass der Fokus hier auf der Regionalplanung liegt und dabei insbesondere die (Planungs-)Regionen, die Träger der Regionalplanung sowie deren Organisation vergleichend unter die Lupe genommen werden. Gerade die Ebene der Regionalplanung bietet hier ein weites Feld, da sie, wie der Autor einleitend feststellt, in 12 Bundesländern mit insgesamt 107 Planungsregionen und unterschiedlichsten Organisationsformen sehr vielfältig ist. Außerdem treffen gerade auf dieser Planungsebene staatliche und kommunale Planungsvorstellungen - teilweise unvereinbar - aufeinander.

Die vorliegende Arbeit wurde vom Fachbereich Rechtswissenschaft der Universität Konstanz im Wintersemester 2014/2015 als Dissertation bei Prof. Dr. Dr. h. c. Martin Ibler angenommen und befindet sich im Wesentlichen auf dem Stand vom Frühjahr 2014. Mit 450 Seiten reinem Text weist sie auch für ein rechtswissenschaftliches Werk einen beträchtlichen Umfang auf, der durchaus zusammen mit dem Literaturverzeichnis (58 Seiten) auf eine gründliche und detailscharfe Bearbeitung des Themas schließen lässt.

Nach einer Einleitung, die sehr gut in das Thema einführt, widmet sich der Autor im zweiten Teil der Arbeit der Stellung der Regionalplanung im Raumplanungsrecht. Es werden dabei insbesondere die zentralen Begriffe „,(Planungs) Region“, ,Regionalplanung“ und „Träger der Regionalplanung" definiert und gut nachvollziehbar erklärt. Die Ausführungen werden auch durch eine Darstellung der ge- 
schichtlichen Entwicklung der Regionalplanung untermauert. Hier wird die Koordinierungsfunktion der Regionalplanung im System der Raumplanung im Verhältnis zur Landesplanung, aber auch zur kommunalen Bauleitplanung sowie zu den Fachplanungen herausgearbeitet. Beim ,Träger der Regionalplanung“ differenziert der Autor deutlich zwischen den Stellen, die für die Aufstellung eines Regionalplans zuständig sind, und den voll rechtsfähigen Verwaltungsträgern, denen der Regionalplan beispielsweise im Rahmen des Normenkontrollverfahrens zugerechnet wird. Davon unterschieden wird dann die Frage nach der regionalplanerischen Planungshoheit.

Im dritten Teil der Arbeit werden die Rechtsgrundlagen der Regionalplanung erläutert. Die Grundlage dafür legen nach einem kurzen Abschnitt zur Rahmengesetzgebungskompetenz des Bundes bis 2006 die Ausführungen (S. 83-103) zur Änderung der Gesetzgebungskompetenz des Bundes durch die Föderalismusreform I hin zu einer konkurrierenden Gesetzgebungskompetenz gemäß Art. 74 Abs. 1 Nr. 31 GG. Wichtig für die weitere Themenbearbeitung sind hier sicherlich die ergänzenden Ausführungen zur erweiterten Organisationsgewalt des Bundes sowie zur Abweichungsgesetzgebungskompetenz der Länder, in diesem Falle gemäß Art. 84 Abs. 1 GG. Im Hinblick auf den späteren Arbeitsschwerpunkt hätte man sich hier vielleicht noch eine etwas ausführlichere Darstellung der Abgrenzungsprobleme im Kompetenzgefüge zwischen Bund und Ländern gewünscht. Sodann werden auf rund 100 Seiten die einfachgesetzlichen Regelungen von Bund (Raumordnungsgesetz 2009) und den Ländern erläutert. Der Autor legt dabei einen Schwerpunkt auf den Umgang der einzelnen Bundesländer mit ihrer Abweichungsgesetzgebungskompetenz und zeigt mit Blick auf die folgenden Ausführungen - trotz vorherrschender Unklarheiten in der Rechtswissenschaft und mangelnder richtungsweisender Rechtsprechung des Bundesverfassungsgerichts in diesem Bereich - eindeutig die Grenzen der Abweichungsgesetzgebungskompetenz der Länder auf. Er unterscheidet dafür erfreulicherweise klar zwischen ergänzenden und abweichenden Regelungen im Landesrecht. Hangst erkennt dabei zutreffend, dass die Föderalismusreform I nicht unbedingt zu einer erleichterten Rechtsanwendung gegenüber den früheren Problemen mit der Rahmengesetzgebungskompetenz geführt hat. Ebenfalls bekennt der Autor zu Recht, dass die Abweichungsmöglichkeit der Länder aus Art. 72 Abs. 3 S. 1 Nr. 4 GG nicht durch einen ungeschriebenen abweichungsfesten Kern beschränkt sein kann. Der ungeschriebene Grundsatz der Bundestreue soll als Schranke der Kompetenzausübung der Länder erst eingreifen, wenn die Länder eine gänzliche Abschaffung der landesweiten Raumordnungs- oder Regionalplanung anstreben. Der Feststellung von Hangst, dass die befürchtete ,Ping-Pong-Gesetzgebung“ bislang ausgeblieben ist, kann allerdings entgegengehalten werden, dass es dafür vielleicht noch $\mathrm{zu}$ früh ist und es abzuwarten bleibt, wie die Länder nun zukünftig auf die anstehende erste größere Novellierung des Raumordnungsgesetzes unter konkurrierender Gesetzgebungszuständigkeit des Bundes nach ihren ersten Erfahrungen mit der Abweichungsgesetzgebungskompetenz reagieren werden.

Der vierte Teil der Arbeit (S. 210-472), mit „Träger der Regionalplanung“ überschrieben, bildet einen weiteren Schwerpunkt der Abhandlung und gibt einen ausführlichen Überblick über die Organisationsformen für die Regionalplanung in den einzelnen Bundesländern. Die anfänglichen Ausführungen aufgreifend, wird dabei jeweils untersucht, wie die Planungsregionen in den einzelnen Ländern, die eine Regionalplanung aufweisen, gegliedert sind, wer die Planungsträger sind, welche unterschiedlichen Organkompetenzen bei der Aufstellung eines Regionalplans bestehen und welche Organisationsform in den einzelnen Ländern nach der Föderalismusreform gewählt wurde. Unter Bezugnahme auf den vorausgegangenen Teil der Abhandlung werden, für den Leser hilfreich, einzelne Normen klar als Abweichungsgesetzgebung einsortiert. In einem eigenen gleichgewichtigen Abschnitt des vierten Teils werden sodann die Träger der Regionalplanung speziell in Baden-Württemberg, 12 Regionalverbände, unter besonderer Hervorhebung des Verbands Region Stuttgart als StadtUmland-Verband sowie des Regionalverbands Donau-Iller und Verband Region Rhein-Neckar als Landesgrenzen überschreitende Verbände, nach demselben Schema untersucht. Es wird einleuchtend verdeutlicht, dass sich Baden-Württemberg aufgrund der unterschiedlich gewählten Organisationsformen innerhalb eines Bundeslandes besonders gut eignet für eine vertiefte Untersuchung. Das Werk liefert eine für Wissenschaft und Praxis gleichermaßen wertvolle Analyse der vollkommen unterschiedlichen Regionalplanungssysteme und -ansätze im Bundesgebiet und kann hier durchaus langfristig als umfassendes Nachschlagewerk dienen. Sowohl für die Regionalplanung in Baden-Württemberg als auch für alle übrigen Bundesländer mit einer Regionalplanung werden im jeweils zweiten Kapitel abschließende Vergleiche und eine eigene Kategorisierung vorgenommen, die wesentlich zum besseren Verständnis der unterschiedlichen Organisationsformen beitragen. Hangst weist dabei für fast alle Planungsverbände in Baden-Württemberg eine besondere eigene regionalplanerische Planungshoheit nach. Für die anderen Bundesländer erkennt der Autor, dass, abgesehen von Schleswig-Holstein, immer eine zumindest mittelbar demokratisch legitimierte Versammlung den Regionalplan beschließt.

Am Ende der Arbeit findet sich eine mit 12 Seiten bemerkenswert kurze, dafür aber umso prägnantere und gut verständliche Zusammenfassung. Die Untersuchung ist deutlich gegliedert und in einem sehr gut verständlichen Sprachstil geschrieben. Der Übersichtlichkeit auch sehr zuträglich sind die ausführliche Gliederung und besonders das umfangreiche und detaillierte Sachverzeichnis. 